\title{
Zernike-Bessel representation and its application to Hankel transforms
}

\author{
Charles Cerjan \\ Lawrence Livermore National Laboratory, 7000 East Avenue, Livermore, California 94550, USA
}

Received October 27, 2006; revised January 3, 2007; accepted January 5, 2007; posted January 10, 2007 (Doc. ID 76491); published May 9, 2007

\begin{abstract}
The duality between the well-known Zernike polynomial basis set and the Fourier-Bessel expansion of suitable functions on the radial unit interval is exploited to calculate Hankel transforms. In particular, the Hankel transform of simple truncated radial functions is observed to be exact, whereas more complicated functions may be evaluated with high numerical accuracy. The formulation also provides some general insight into the limitations of the Fourier-Bessel representation, especially for infinite-range Hankel transform pairs. () 2007 Optical Society of America

OCIS codes: $000.4430,050.1960,070.2590,100.3020,100.6890$.
\end{abstract}

\section{INTRODUCTION}

Hankel transforms naturally arise from the evaluation of a two-dimensional Fourier transform with radial symmetry. Since the imposition of exact or approximate radial geometry is ubiquitous in Fourier-transform-based optical propagation, Hankel transform techniques remain an important analytical and numerical tool in these investigations. More specifically, the integral $p$ th-order Hankel transforms are the coefficients in the radial Fourier decomposition of a given function

$$
\hat{f}(u, v)=\sum_{p=-\infty}^{\infty} i^{p} \hat{f}_{p}(\rho) \exp (i p \phi),
$$

where $u=\rho \cos (\phi), v=\rho \sin (\phi)$ are radial coordinates, $\hat{f}(u, v)$ is the Fourier transform of the given function $f(x, y)$, and

$$
\hat{f}_{p}(\rho)=2 \pi \int_{0}^{\infty} f_{p}(r) J_{p}(2 \pi r \rho) r \mathrm{~d} r
$$

with the usual notation for the integral $p$ th-order Bessel functions, $J_{p}(z) .{ }^{1}$ The inverse transform is of course symmetric:

$$
f_{p}(r)=2 \pi \int_{0}^{\infty} \hat{f}_{p}(\rho) J_{p}(2 \pi \rho r) \rho \mathrm{d} \rho .
$$

Some of the most demanding applications of the Hankel transform occur in computational optical sectioning microscopy, where rapidly varying sweep signals and pupil functions pose severe accuracy and timing challenges for existing numerical techniques. These approaches have been recently reviewed, applied, and, in some cases, extended. ${ }^{2}$ As emphasized in this earlier work, considerable care is required to obtain numerical accuracy from any of the many competing algorithms. The difficulties that remain with the evaluation of this class of highly oscillatory functions, though, motivate the present investigation.
In the method proposed here, the duality between the well-known Zernike polynomials ${ }^{3}$ and the Bessel functions on the radial unit interval is exploited to produce highly accurate expressions for the Hankel transforms of functions truncated on this interval. The duality arises from the observation that a series expansion of suitable functions in terms of Zernike polynomials is simply related to the corresponding Fourier-Bessel series. Since both basis sets are complete, a given function may be expanded in terms of either series and, in particular, a specified-order Bessel function has a convergent series representation in terms of Zernike polynomials that provides an alternate form of the associated Hankel transform. Conversely, the Hankel transform of a polynomial function restricted to the unit interval is represented exactly by the corresponding Bessel function. An interesting numerical aspect of the Zernike and Fourier-Bessel series duality is the utility of the often rediscovered discrete version of the Fourier-Bessel series. ${ }^{4-8}$ In this expansion, the roots of a given-order Bessel function become the quadrature points of the integral evaluation, which has the important numerical property of preserving the inverse Hankel transform to the same accuracy as the direct transform.

In the following section, the general formalism is briefly described. The subsequent sections detail applications of this formalism to the evaluation of Hankel transforms of finite-range functions such as the generalized top-hat functions and their inverses; the highly oscillatory sweep signal and pupil function Hankel transforms in Markham ${ }^{2}$; and the infinite-range Hankel transforms, such as the sinc function. The final section concludes the presentation with a discussion of the limitations of the general formalism, especially with respect to the intrinsically infinite -range Hankel transform.

\section{ZERNIKE-BESSEL REPRESENTATION}

The Zernike polynomials are important in optical physics due to their convenient representation of the various lens- 
diffractive aberration terms. This set of polynomials is specifically constructed to be a complete, orthogonal set on the unit radial interval ${ }^{3}$

$$
\int_{0}^{1} R_{n}^{l}(\rho) R_{n^{\prime}}^{l}(\rho) \rho \mathrm{d} \rho=\frac{1}{2(n+1)} \delta_{n n^{\prime}},
$$

where $R_{n}^{l}(\rho)$ denotes the Zernike polynomial of integer order $n$ and integer degree $l$ as a function of the radial variable $\rho$. The Zernike polynomials are a special case of the Jacobi polynomials, ${ }^{3,9}$

$$
P_{n^{\prime}}^{(\alpha, \beta)}(x)=(-1)^{n^{\prime}} \frac{R_{n}^{l}(\rho)}{\rho^{\alpha}},
$$

where $P_{n^{\prime}}^{(\alpha, \beta)}(x)$ are the standard Jacobi polynomials ${ }^{10}$ with $x=1-2 \rho^{2}, \beta=0, \alpha=l$, and $n^{\prime}=(n-l) / 2$. Since the Zernike polynomials are related directly to the Jacobi polynomials, they possess both three-term recurrence relationships for both degree and order and may be numerically integrated with Gaussian quadrature points and weights. Their oscillatory behavior for large order, however, often complicates their numerical application. ${ }^{9}$

Recall that the Fourier-Bessel series expansion expresses some suitable function, $f(\rho)$, on the radial unit interval in terms of a Bessel function of fixed order, $J_{l}(\rho)$, whose argument is scaled by the positive zeros of that Bessel function, $\alpha_{l m}$,

$$
f(\rho)=\sum_{m=1}^{\infty} \frac{2 c_{m}}{J_{l+1}^{2}\left(\alpha_{l m}\right)} J_{l}\left(\alpha_{l m} \rho\right),
$$

where the coefficients, $c_{m}$, are determined by the overlap integrals

$$
c_{m}=\int_{0}^{1} f(\rho) J_{l}\left(\alpha_{l m} \rho\right) \rho \mathrm{d} \rho .
$$

These coefficients are recognizable as the Hankel transform of order $l$ for the function $f(\rho)$ evaluated at the Bessel function roots. Applying the Fourier-Bessel expansion to a fixed-degree Zernike polynomial produces

$$
R_{n}^{l}(\rho)=2(-1)^{(n-l) / 2} \sum_{m=1}^{\infty} \frac{J_{n+1}\left(\alpha_{l m}\right)}{\alpha_{l m} J_{l+1}^{2}\left(\alpha_{l m}\right)} J_{l}\left(\alpha_{l m} \rho\right),
$$

using the explicit form of the overlap integral in Eq. (7) between Zernike and Bessel functions ${ }^{3}$

$$
\int_{0}^{1} R_{n}^{l}(\rho) J_{l}(k \rho) \rho \mathrm{d} \rho=(-1)^{(n-l) / 2} \frac{J_{n+1}(k)}{k} .
$$

Conversely, using the completeness of the Zernike polynomials, any suitable function may be expanded on this interval as

$$
f(\rho)=\sum_{n=l}^{\infty} 2(n+1) b_{n} R_{n}^{l}(\rho),
$$

where the necessary overlap coefficients are

$$
b_{n}=\int_{0}^{1} f(\rho) R_{n}^{l}(\rho) \rho \mathrm{d} \rho .
$$

Summarizing this development, two expansions, Eqs. (6) and (7), express the function $f(\rho)$ in terms of either the Bessel functions or the Zernike polynomials. The two coefficient sets, $b_{n}$ and $c_{m}$, may be expressed in terms of each other by using the respective orthogonality of the basis sets. That is, starting from

$$
f(\rho)=\sum_{n=l}^{\infty} 2(n+1) b_{n} R_{n}^{l}(\rho)=\sum_{m=1}^{\infty} \frac{2 c_{m}}{J_{l+1}^{2}\left(\alpha_{l m}\right)} J_{1}\left(\alpha_{l m} \rho\right),
$$

multiplying by $R_{n^{\prime}}^{l}(\rho)$, and integrating over the radial unit interval, the coefficients $b_{n}$ are

$$
b_{n}=(-1)^{(n-l) / 2} \sum_{m=1}^{\infty} c_{m} \frac{J_{n+1}\left(\alpha_{l m}\right)}{\alpha_{l m}},
$$

where the coefficients $c_{m}$ are the Hankel transforms evaluated at the Bessel zeros. Correspondingly, the coefficients $c_{m}$ may be expressed in the form

$$
c_{m}=J_{l+1}^{2}\left(\alpha_{l m}\right) \sum_{n=l}^{\infty}(-1)^{(n-l) / 2} 2(n+1) b_{n} \frac{J_{n+1}\left(2 \pi \alpha_{l m}\right)}{2 \pi \alpha_{l m}} .
$$

For example, specializing to the case of $f(\rho)=J_{l}(k \rho)$ produces an interpolation formula for a given Bessel function in terms of Zernike polynomials

$$
J_{l}(k \rho)=\sum_{n=l}^{\infty}(-1)^{(n-l) / 2} 2(n+1) \frac{J_{n+1}(k)}{k} R_{n}^{\prime}(\rho),
$$

with the specific choice $k=\alpha_{l m}$ yielding

$$
J_{l}\left(\alpha_{l m} \rho\right)=\sum_{n=l}^{\infty}(-1)^{(n-l) / 2} 2(n+1) \frac{J_{n+1}\left(\alpha_{l m}\right)}{\alpha_{l m}} R_{n}^{\prime}(\rho) .
$$

This last series expansion displays the expected duality between the Zernike polynomial basis set and the Fourier-Bessel basis set. It is also interesting to note that these expressions provide an alternate polynomial expansion of an integral-order Bessel function.

Likewise, a few special cases of these series expressions may be noted. When $n=l \neq 0$, the Zernike polynomial becomes a monomial, $R_{n}^{n}(\rho)=\rho^{n}$, and Eq. (8) reduces to

$$
\rho^{n}=2 \sum_{m=1}^{\infty} \frac{J_{n}\left(\alpha_{n m} \rho\right)}{\alpha_{n m} J_{n+1}\left(\alpha_{n m}\right)},
$$

which is a well-known representation. ${ }^{11}$ Thus the generalorder top-hat function may be expressed in a FourierBessel series that will be immediately relevant to the Hankel transform applications below. Although this series representation is uniformly convergent for $0 \leqslant \rho<1$, it does not converge at $\rho=1$, which is simply verified by inserting this value and obtaining a contradiction. Of course the series will be numerically poorly behaved near this end point-an aspect of the Fourier-Bessel expansion that is often overlooked. This deficiency is easily remedied by applying the Dini series representation ${ }^{12}$ to obtain a uniformly convergent expansion for the entire interval with consequent superior numerical performance. This point will be revisited below. 
Some simple summation formulas, relevant to discrete Hankel transform applications, follow from Eq. (8). Specifically, by multiplying this expression by $R_{n^{\prime}}^{l}(\rho)$, integrating over the radial interval, and using the orthogonality of the Zernike polynomials, the equality

$$
\frac{\delta_{n n^{\prime}}}{2(n+1)}=2 \sum_{m=1}^{\infty} \frac{J_{n+1}\left(\alpha_{l m}\right) J_{n^{\prime}+1}\left(\alpha_{l m}\right)}{\alpha_{l m}^{2} J_{l+1}^{2}\left(\alpha_{l m}\right)}
$$

arises. The special case $n=n^{\prime}=l$ leads to

$$
\frac{1}{4(n+1)}=\sum_{m=1}^{\infty} \frac{1}{\alpha_{n m}^{2}} \text {. }
$$

Summation expressions of this type might be useful to estimate the number of series terms needed for a requested level of numerical accuracy.

\section{FINITE-RANGE TRANSFORMS}

The Bessel-Zernike representation provides insight into the evaluation of finite-range transforms; that is, the Hankel transform of a function that is exactly truncated on the unit radial interval. A simple example would be the generalized "top-hat" function defined as $f_{p}(r)=r^{p}(H(r)$ $-H(r-1))$, where $H(r)$ is the Heaviside step function. As noted above, the monomial $r^{p}$ is just the particular Zernike polynomial with $n=l=p$, so its Hankel transform is immediately obtained from Eq. (9):

$$
\hat{f}_{p}(\rho)=\frac{J_{p+1}(\rho)}{\rho},
$$

which is, of course, the exact value. An examination of the inverse Hankel transform reveals a connection with the discretization technique used in the quasi-discrete Hankel transform representation. ${ }^{5,8}$ Consider the FourierBessel series coefficients of Eq. (7) in this case,

$$
c_{m}=\int_{0}^{1} \frac{J_{p+1}(\rho)}{\rho} J_{p}\left(\alpha_{p m} \rho\right) \rho \mathrm{d} \rho,
$$

where the original infinite interval has been suitably truncated and scaled to the unit interval. Approximating these overlap coefficients by

$$
c_{m} \approx \frac{J_{p+1}\left(\alpha_{p m}\right)}{\alpha_{p m}}
$$

and inserting them into Eq. (6) produces

$$
f_{p}(r) \approx 2 \sum_{m=1}^{\infty} \frac{J_{p}\left(\alpha_{l m} r\right)}{\alpha_{p m} J_{p+1}\left(\alpha_{l m}\right)}
$$

which is recognized as an approximation of the original pth-order Zernike monomial in Eq. (17).

As mentioned previously, the Fourier-Bessel series representation is uniformly convergent on the interval 0 $\leqslant r<1$ and obviously fails at $r=1$. Numerically, the summation must be truncated and convergence will be poor in this neighborhood. A modification of this series, due to Dini, ${ }^{11,12}$ leads to uniform convergence over the entire in- terval and much-improved numerical behavior near $r=1$. Briefly, the Fourier-Bessel representation of Eq. (6) is replaced by

$$
f(\rho)=\sum_{m=1}^{\infty} 2 d_{m} J_{l}\left(\beta_{l m} \rho\right),
$$

where the Dini coefficients are

$$
\begin{aligned}
d_{m}= & \beta_{l m}^{2}\left\{\beta_{l m}^{2}\left[J_{l}^{\prime}\left(\beta_{l m}\right)\right]^{2}+\left(\beta_{l m}^{2}-l^{2}\right)\right. \\
& \left.\times\left[J_{l}\left(\beta_{l m}\right)\right]^{2}\right\}^{-1} \int_{0}^{1} t J_{l}\left(\beta_{l m} t\right) f(t) \mathrm{d} t,
\end{aligned}
$$

and the values $\beta_{l m}$ are the positive roots of $t J_{l}^{\prime}(t)+a J_{l}(t)$ $=0$. There is some flexibility in the choice of the $\beta_{l m}$ since the value of $a$ is somewhat arbitrary. For example, the choice $a+l=0$ leads to the condition $J_{l+1}\left(\beta_{l m}\right)=0$, and the $\beta_{l m}$ are no more difficult to obtain than the original Fourier-Bessel roots. However, this choice must be accompanied by an additional term to handle a pole at the origin, ${ }^{12}$ which might be numerically difficult to evaluate in general cases. This problem may be avoided by choosing $a=0$ so that the values are determined by $J_{l}^{\prime}\left(\beta_{l m}\right)=0,{ }^{13}$ which also simplifies the denominator in Eq. (25). As an example, the Fourier-Bessel series of Eq. (17) may be compared with the analogous Dini series, which has the explicit form

$$
\rho^{n}=2 \sum_{m=1}^{\infty} \frac{\beta_{n m} J_{n+1}\left(\beta_{n m}\right) J_{n}\left(\beta_{n m} \rho\right)}{\left(\beta_{n m}-n^{2}\right) J_{n}^{2}\left(\beta_{n m}\right)} .
$$

Numerically, this series representation is superior to the Fourier-Bessel expression of Eq. (17). For the specific, arbitrary choice of $n=3$, the logarithm of the absolute difference between the exact function, $r^{3}$, and the two series representations is plotted in Fig. 1 when both series are truncated to 512 terms. The error associated with the Dini series is approximately 3 orders of magnitude smaller than the Fourier-Bessel error over the entire interval and, as expected, is much more accurate at the end point.

An especially challenging finite-range Hankel transform has been identified by Markham and Conchello ${ }^{2}$ in

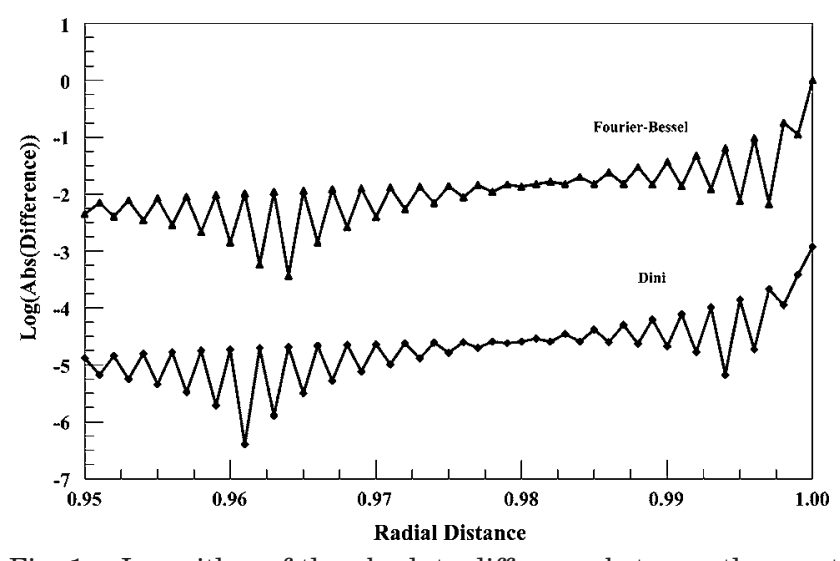

Fig. 1. Logarithm of the absolute difference between the exact result and the Fourier-Bessel series (upper curve) and the Dini series (lower curve) plotted near the unit interval end point. 


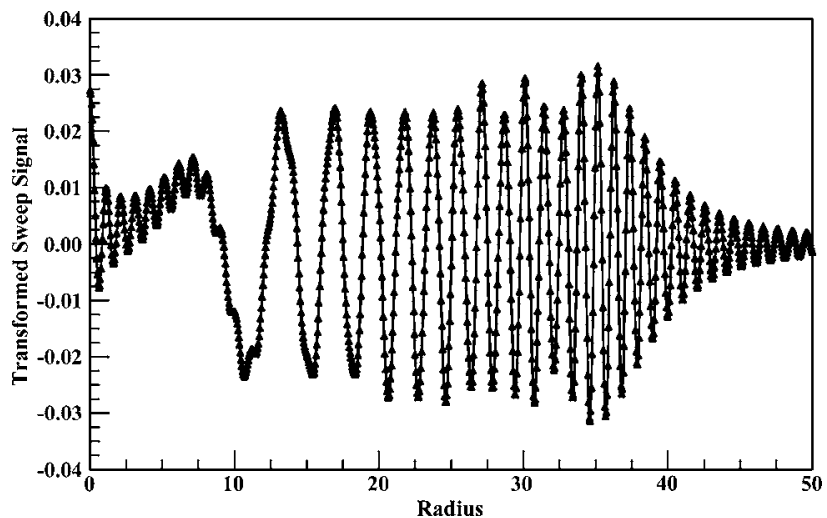

Fig. 2. Evaluation of the Hankel transform of the sweep signal in the Zernike-Bessel representation retaining 150 terms in the summation.

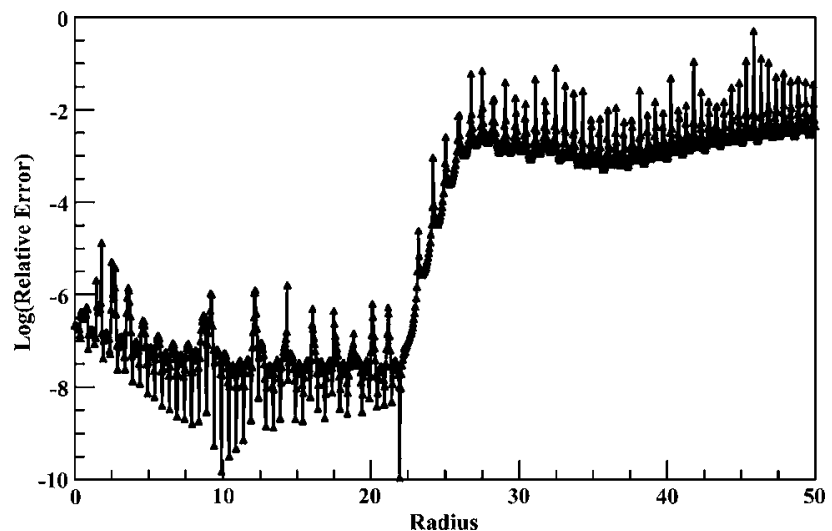

Fig. 3. Logarithm of the absolute value of the relative error between the Gauss-Kronrod and the Zernike-Bessel evaluations for 80 terms for the Hankel transform of the sweep function.

their consideration of the numerical evaluation of the zero-order transform of an oscillating sweep signal with the functional form

$$
f(r)=\sin \left(\frac{\pi d}{b-a}\left\{\left[\frac{r(b-a)}{d}+a\right]^{2}-a^{2}\right\}\right),
$$

where $a=5, b=40$, and $d=1$ on the unit radial interval; the function vanishes for $r>1$. Applying Eqs. (10) and (11) to evaluate the zero-order transform leads to

$$
\hat{f}_{0}(r)=2 \pi \sum_{n=0}^{\infty}(-1)^{n / 2} \frac{J_{n+1}(2 \pi r)}{2 \pi r} 2(n+1) \int_{0}^{1} f(\rho) R_{n}^{0}(\rho) \rho \mathrm{d} \rho,
$$

which reexpresses the transformed function as a series of Bessel functions, thus accounting for the observed complicated interference structure observed previously. From a numerical perspective, this series representation is competitive with Gauss-Kronrod quadrature. More specifically, truncating the series at 150 terms and evaluating the Zernike overlap integrals by Simpson's rule with a $\Delta r$ spacing of $2.5 \times 10^{-4}$, the transform appears as in Fig. 2 for $\rho \leqslant 50$. The agreement with the Gauss-Kronrod evaluation is quantitative as demonstrated in Figs. 3-5 for different series truncation values. In these figures, the logarithm of the absolute value of the relative error. $\mid\left(f_{G K}\right.$ $\left.-f_{\text {Zernike }}\right) / f_{G K} \mid$, is plotted for $n=80,120$, and 150 . Since the $n$ th-order Bessel function vanishes as $\rho^{n}$ at the origin, the higher-order summation terms contribute only at successively larger $\rho$ values; hence convergence near the origin occurs within the first few terms. Note that no terms need to be recalculated for this comparison and that arbitrary $\rho$ values could be chosen since the series solution is essentially a uniform fit of the Hankel transform to Bessel functions over the unit interval. Also, the Bessel functions may be evaluated to very high accuracy, so the residual error is probably dominated by the evaluation of the Zernike overlap integrals.

In the same vein, Markham and Conchello ${ }^{2}$ considered the propagation of an out-of-focus pupil function using the optical path difference (OPD) derived by Gibson and Lanni. ${ }^{14}$ The real part of this pupil function at a distance of $0.01575 \mathrm{~mm}$ (propagation plane 63) is plotted in Fig. 6 . Applying the Zernike-Bessel expansion, the zeroth-order Hankel transform is readily evaluated and is displayed in Fig. 7 for 120 terms in the summation. The agreement with Gauss-Kronrod quadrature is shown in Fig. 8, where the logarithm of the absolute value of the relative error is again plotted in transform coordinates. All of the convergence trends noted for the case of the sweep signal obtain. The relative accuracy is high and improves at larger distances with more summation terms.

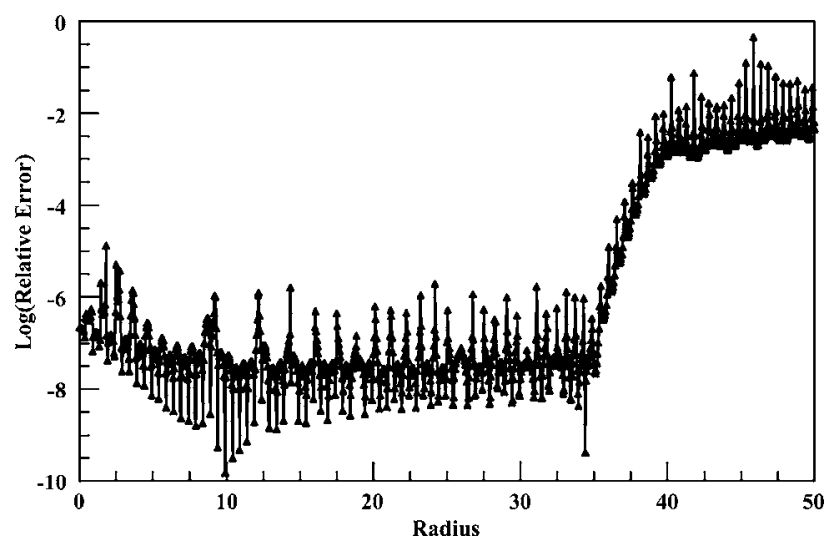

Fig. 4. Logarithm of the absolute value of the relative error between the Gauss-Kronrod and the Zernike-Bessel evaluations for 120 terms for the Hankel transform of the sweep function.

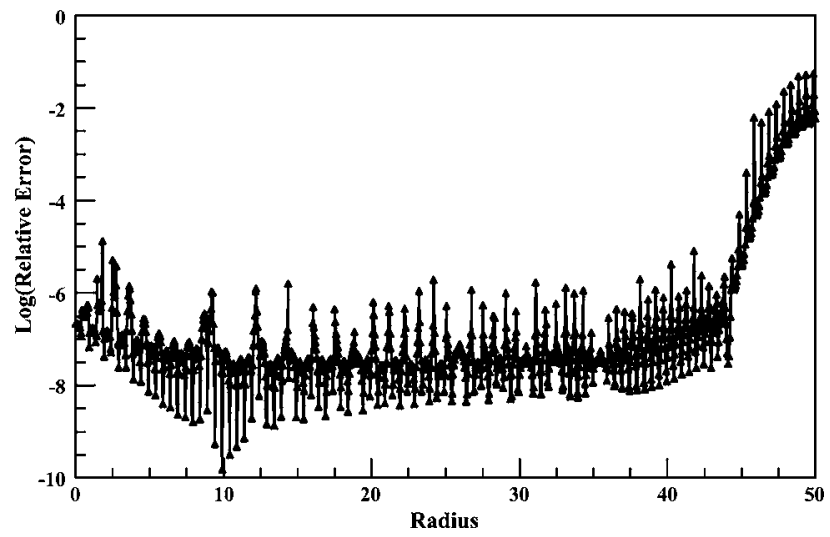

Fig. 5. Logarithm of the absolute value of the relative error between the Gauss-Kronrod and the Zernike-Bessel evaluations for 150 terms for the Hankel transform of the sweep function. 


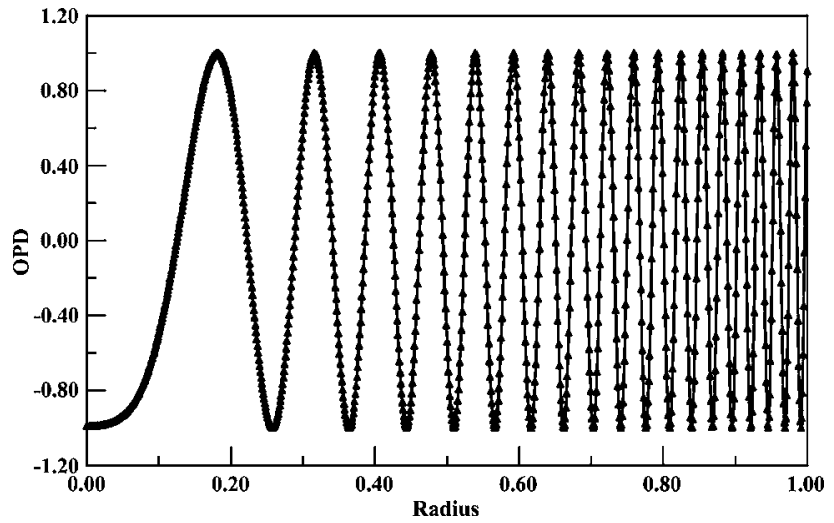

Fig. 6. Real part of the out-of-focus pupil function at a propagation distance of $0.01575 \mathrm{~mm}$ derived from Eq. (4) of Gibson and Lanni. ${ }^{14}$



Fig. 7. Evaluation of the Hankel transform of the pupil function in the Zernike-Bessel representation retaining 120 terms in the summation.

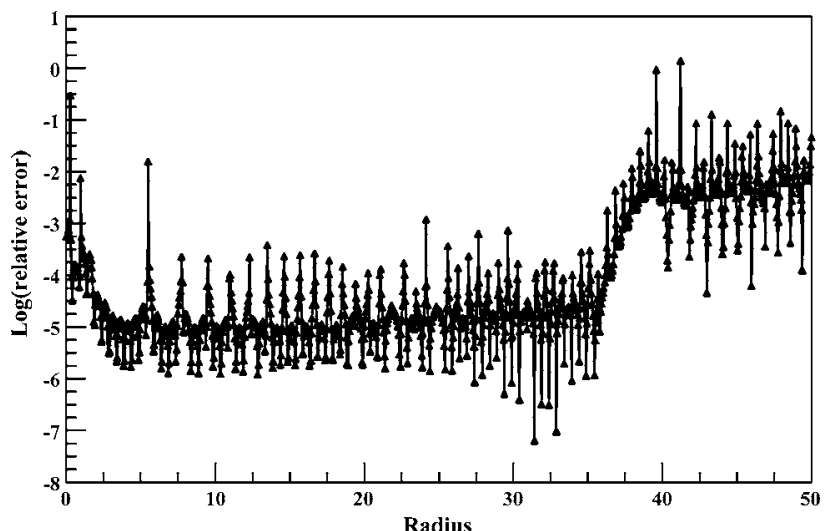

Fig. 8. Logarithm of the absolute value of the relative error between the Gauss-Kronrod and the Zernike-Bessel evaluations for 120 terms for the Hankel transform of the pupil function.

The Zernike-Bessel expansion may also be used to evaluate the quasi-discrete Hankel transform ${ }^{5,8}$ for the sweep function noted above. Specifically, since the Zernike-Bessel series provides a uniformly convergent representation at all points of the open radial unit interval, the quasi-discrete Hankel transform may be obtained by evaluating the series at the scaled zeros of the appropriate Bessel function and forming the Fourier-Bessel series. In the particular example of the sweep function dis- cussed above, the quasi-discrete zeroth-order Hankel transform appears in Fig. 9 using 256 mesh points. The logarithm of the absolute value of the relative error is plotted in Fig. 10, while the same relative error at 512 mesh points is plotted in Fig. 11. Although the overall functional form is captured relatively well even at the



Fig. 9. Quasi-discrete zeroth-order Hankel transform of the sweep function in Fig. 2 using 256 mesh points and a 120 -term Zernike-Bessel representation plotted as a function of radial variable.

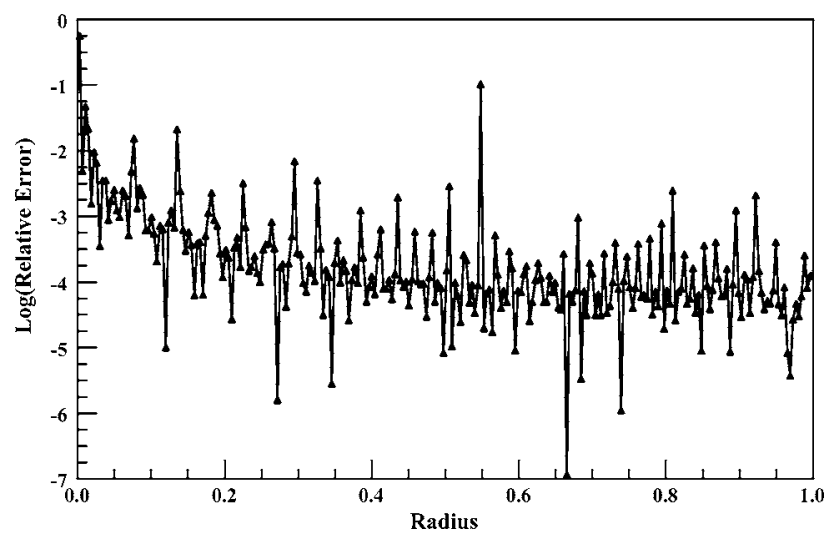

Fig. 10. Logarithm of the absolute value of the relative error between the exact transform of the sweep function and the 256point quasi-discrete zeroth-order Hankel transform as a function of radial variable.

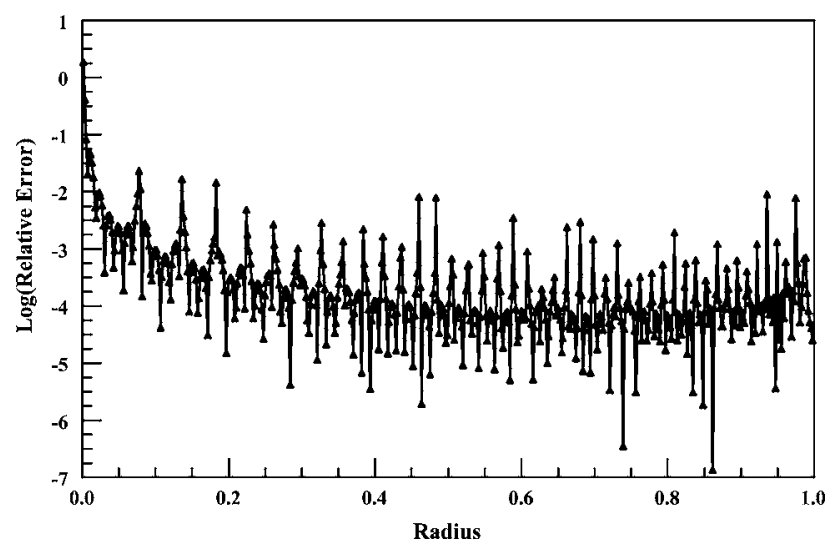

Fig. 11. Logarithm of the absolute value of the relative error between the exact transform of the sweep function and the 512point quasi-discrete zeroth-order Hankel transform as a function of radial variable. 
smaller number of mesh points, there is a persistent error near the origin due to the conditional convergence of the Fourier-Bessel series. That is, the truncated quasidiscrete Hankel series in Eq. (6) for $l=0$ evaluated near or at the origin provides a poor approximation of the value of the exact Hankel transform, which vanishes. The error becomes worse for a larger number of mesh points since a region closer to the origin is sampled for 512 points compared with 256 points.

\section{INFINITE-RANGE TRANSFORMS}

In the cases considered above, favorable series convergence was noted for those functions strictly represented on the unit interval, whereas the infinite-range transform might be more difficult to represent accurately, especially when the exact transform integral depends sensitively upon cancellation of the oscillations at infinity. A striking example of the limitations encountered by the series representation of transform pairs that are both of infinite extent is provided by the sinc function

$$
f(r)=\frac{\sin (2 \pi \gamma r)}{2 \pi \gamma r}
$$

and its $p$ th-order Hankel transform

$$
\hat{f}(\rho)=\left\{\begin{array}{lc}
\frac{\rho^{p} \cos (p \pi / 2)}{2 \pi \gamma \sqrt{\gamma^{2}-\rho^{2}}\left(\gamma+\sqrt{\gamma^{2}-\rho^{2}}\right)^{p}} & \text { if } 0 \leq \rho \leq \gamma \\
\frac{\sin [p \arcsin (\gamma / \rho)]}{2 \pi \gamma \sqrt{\rho^{2}-\gamma^{2}}} & \text { if } \rho>\gamma
\end{array} .\right.
$$

The accuracy of the Fourier-Bessel representation of this pair of functions has been recently considered in the context of the quasi-discrete Hankel transform for orders $p$ $=1$ and 4 , where the stability of the unitary transformation between the functions is demonstrated. ${ }^{8}$ However, it should be noted that both the Fourier-Bessel and Zernike-Bessel series representations for the sinc function are poorly behaved at the origin. In particular, either series representation vanishes for a finite number of terms, whereas the exact function approaches unity at the origin. This effect is apparent in Fig. 12, which contains a plot of the logarithmic absolute error between the Zernike-Bessel approximation and the exact sinc function for $\gamma=5$. As expected, the error is largest near the origin, but the relative error is large everywhere, indicating the poor quality of the fit over the plotted interval. Since the $p$ th-order Hankel transform vanishes at the origin, the overall error introduced by either the Fourier-Bessel or the Zernike-Bessel series representation of the first-order Hankel transform is not as severe, as shown by the logarithmic error plots in Figs. 13 and 14, respectively, for the different series. The error is concentrated near the singularity since a continuous series representation cannot reproduce an exact discontinuity. The difficulties associated with this demanding transform pair highlight the limitations of truncating intrinsically infinite functions to the unit interval, since the existence of the exact integral formulation depends upon cancellation of oscillating terms

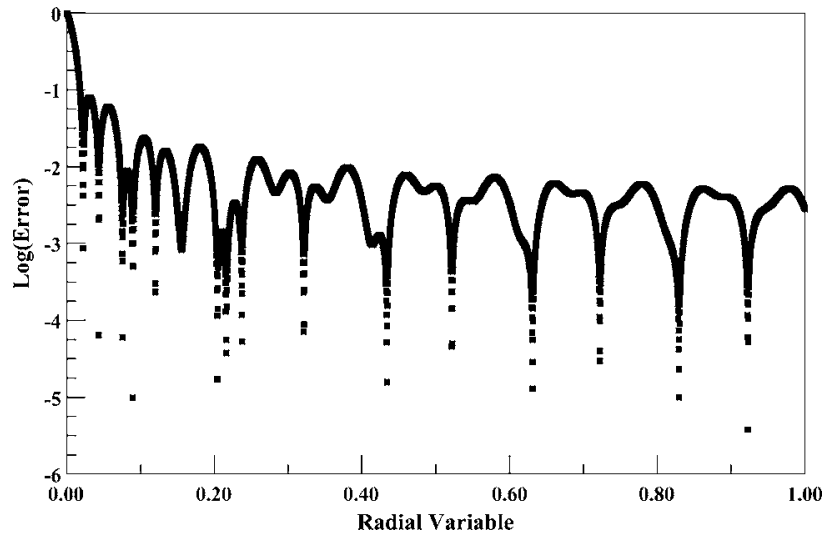

Fig. 12. Logarithm of the absolute value of the difference between the sinc function and the 150-term Zernike-Bessel representation plotted as a function of radial variable.

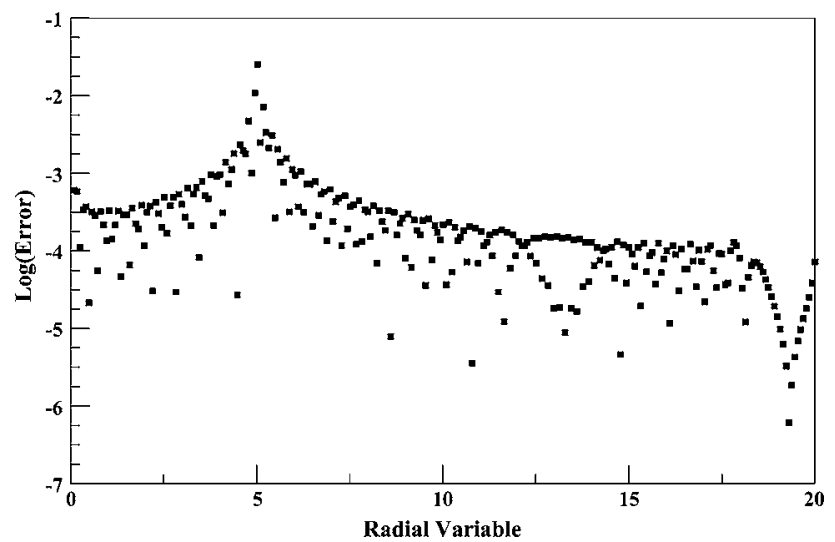

Fig. 13. Logarithm of the absolute value of the difference between the exact transform of the sinc function and the 256-point quasi-discrete first-order Hankel transform as a function of radial variable.

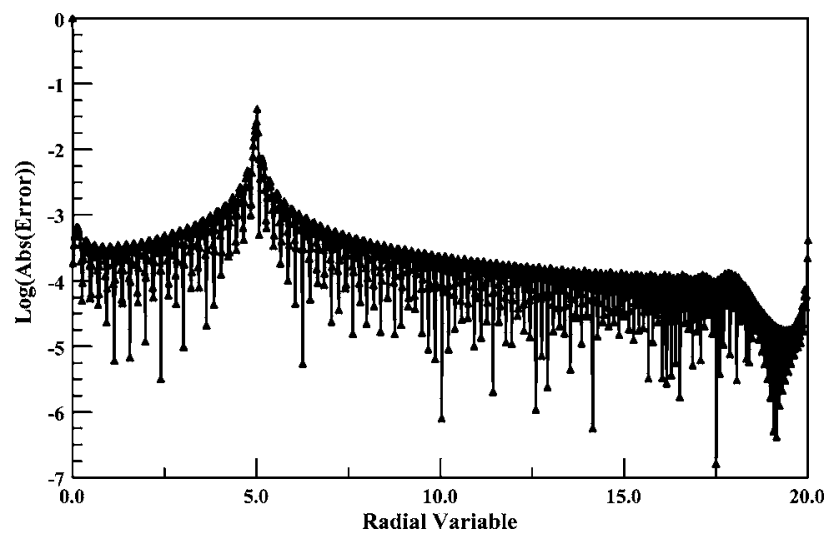

Fig. 14. Logarithm of the absolute value of the difference between the exact transform of the sinc function and the 150-term Zernike-Bessel representation of the first-order Hankel transform plotted as a function of radial variable.

in the integrand at large radial distances. Functions that are better behaved at large distances, for example, exponentially bounded integrands, will probably not suffer from these inaccuracies.

\section{CONCLUSION}

Briefly, the Zernike-Bessel series has several interesting theoretical and practical aspects. The algorithmic imple- 
mentation follows directly from Eqs. (10) and (11): The overlap integrals of a given test function are evaluated, and the resulting series representation is truncated to some desired accuracy. This approach appears to be most accurate and numerically useful for functions that are strictly confined to the unit interval where it obtains an accuracy competitive with the most expensive adaptive quadiature scheme and, consequently, an accuracy much higher than that of standard methods. Since the overlap integrals need only be computed once and stored, the resulting series evaluation may be very efficient upon repeated evaluation. Also, the Zernike-Bessel series shares many of the positive features of the discrete FourierBessel approach since it is directly related to this particular representation. Superior accuracy may also be achieved in some circumstances by the use of the Dini series form, which eliminates end-point singularities. On the other hand, numerical difficulties are sometimes encountered with conditionally convergent, infinite-range transform pairs. Some classes of transform pairs depend sensitively upon asymptotic cancellation for convergence so that the resulting series summation requires many terms. Infinite-range integrals that decay more quickly, such as exponentials, though, do not suffer from this difficulty and will require far fewer expansion terms. Another practical difficulty with the direct implementation of the series is the evaluation of the overlap integrals between the given function and the Zernike polynomials.

Expanding upon this point, the Zernike polynomials are well known to be highly oscillatory and thus subject to numerical instability for high-order evaluation. Although direct calculation of the overlap integrals with high precision or Gaussian quadrature is possible, the asymptotic expansion of the Jacobi polynomials offers an alternative approach for the evaluation of the product of high-order Zernike polynomials with a given test function. More specifically, in the limit $n \rightarrow \infty$ this asymptotic expansion is $^{15,16}$

$$
\begin{aligned}
P_{n}^{(\alpha, \beta)}(\cos \theta) \approx & \frac{\cos \left[\left(n+\frac{\alpha+\beta+1}{2}\right) \theta-(\pi / 4)(1+2 \alpha)\right]}{\sqrt{\pi n}[\sin (\theta / 2)]^{1 / 2+\alpha}[\cos (\theta / 2)]^{1 / 2+\beta}} \\
& +O\left(n^{-3 / 2}\right),
\end{aligned}
$$

for $0<\theta<\pi$. Since the Zernike polynomials are simply related to the general Jacobi polynomials by Eq.(5), applying this expansion is straightforward. Even though the expansion fails near the end points, if the overlap function in the integrand itself vanishes at the end points, then no special care is required to successfully calculate the integrals. The evaluation of the Zernike-Bessel coefficients for the sweep function above was tested for $n$ $>80$, and good agreement with the direct evaluation of the coefficients was observed even with Simpson's rule integration. Inexpensive quadrature techniques of course greatly improve the numerical efficiency. In general, though, the evaluation of the Zernike-Bessel coefficients will present a numerical challenge in the practical application of this series.

In summary, the primary intent of the above discussion is the introduction of the Zernike-Bessel representation as a complementary series expression to the better-known Fourier-Bessel series. The duality between the series is a consequence of the simple overlap integral between the Zernike polynomials and a Bessel function on the unit radial interval. It was noted that the Zernike-Bessel representation is not limited to special mesh values, such as the zeros of the Bessel functions, and may be used to fit suitable functions to a sum of Bessel functions on the unit interval and that truncated polynomials possess an exact series representation. Additionally, the Fourier-Bessel series is known to be uniformly convergent on the open unit interval with possible divergence at the end points, but a particular choice of Dini series will overcome this limitation. The connection between the Zernike-Bessel series evaluation of a Hankel transform and the quasi-discrete approach is clear. Both representations suffer convergence failures in some infinite-range cases such as the sinc function, but in other, more favorable cases, the Zernike-Bessel series might possess computational advantages. Finally, the Zernike-Bessel representation might offer further insight into the properties of the Hankel transform, as exemplified by the sweep function above.

\section{ACKNOWLEDGMENTS}

This work was performed under the auspices of the U.S. Department of Energy by the University of California, Lawrence Livermore National Laboratory under contract W-7405-Eng-48.

Charles Cerjan can be reached by e-mail at cerjan1@llnl.gov.

\section{REFERENCES AND NOTES}

1. G. E. Andrews, R. Askey, and R. Roy, Special Functions (Cambridge U. Press, 1999), pp. 210-216.

2. J. Markham and J.-A. Conchello, "Numerical evaluation of Hankel transforms for oscillating functions," J. Opt. Soc. Am. A 20, 621-630 (2003).

3. M. Born and E. Wolf, Principles of Optics, 6th ed. (Cambridge U. Press, 1980), Section 9.2 and Appendix VII.

4. F. Lado, "Equation of state of the hard-disk fluid from approximate integral equations," J. Chem. Phys. 49, 3092-3096 (1968).

5. H. Fisk Johnson, "An improved method for computing the discrete Hankel transform," Comput. Phys. Commun. 43, 181-202 (1987).

6. D. Lemoine, "The discrete Bessel transform algorithm," J. Chem. Phys. 101, 3936-3984 (1994).

7. L. Yu, M. Huang, M. Chen, W. Chen, W. Huang, and Z. Zhu, "Quasi-discrete Hankel transform," Opt. Lett. 23, 409-411 (1998).

8. M. Guizar-Sicairos and J. C. Gutiérrez-Vega, "Computation of quasi-discrete Hankel transforms of integer order for propagating optical wave fields," J. Opt. Soc. Am. A 21, 53-58 (2004).

9. A. Prata, Jr., and W. V. T. Rusch, "Algorithm for computation of Zernike polynomials expansion coefficients," Appl. Opt. 28, 749-754 (1989).

10. A. Abramowitz and I. Stegun, eds., Handbook of Mathematical Functions (U. S. Government Printing Office, 1972), Chap. 22.

11. W. Magnus, F. Oberhettinger, and R. P. Soni, Formulas and 
Theorems for the Special Functions of Mathematical Physics (Springer-Verlag, 1966), p. 128.

12. G. N. Watson, A Treatise on the Theory of Bessel Functions (Cambridge U. Press, 1966), Chap. XVIII.

13. The computer package, MATHEMATICA (Wolfram Research, Inc.), conveniently includes an algorithm for the roots of the derivatives of the Bessel functions.

14. F. S. Gibson and F. Lanni, "Experimental test of an analytical model of aberration in an oil-immersion objective lens used in three-dimensional light microscopy," J. Opt. Soc. Am. A 8, 1601-1613 (1991).

15. W. Magnus, F. Oberhettinger, and R. P. Soni, Formulas and Theorems for the Special Functions of Mathematical Physics (Springer-Verlag, 1966), p. 216.

16. I. S. Gradshteyn and I. M. Ryzhik, Table of Integrals, Series, and Products, 4th ed. (Academic, 1980), pp. 1037, 8.965 . 